\title{
MEALINESS ASSESSMENT IN APPLES USING MRI TECHNIQUES
}

\author{
P. Barreiro, J. Ruiz-Cabello, M.E. Fernández-Valle, C. Ortiz, and M. Ruiz-Altisent \\ Departamento de Ingenieria Rural, ETSLA and Unidad de RMN, UCM. 28040, Madrid, Spain
}

\begin{abstract}
Small samples of Top Red apples stored 6 months under controlled atmosphere (expected to be non-mealy) and $2^{\circ} \mathrm{C}$ (expected to be mealy) have been used for MRI imaging. Multi-slice-multi-echo magnetic resonance images (64*64 pixels) have been recorded with a $8 \mathrm{~ms}$ echo time. Three out of four apples corresponding to the sample maintained under controlled atmosphere did not developed mealiness while three out of four fruits corresponding to the sample stored at $2^{\circ} \mathrm{C}$ became mealy after 6 months of storage. The minimum $T_{2}$ values obtained for the mealy apples show to be significantly lower $(F=13.21)$ when compared with non-mealy apples pointing that a more desegregated structure and a lower juiciness content leads to lower $T_{2}$ signal. Also, there is a significant linear correlation $\left(r=-0.76\right.$ ) between the number of pixels with a $T_{2}$ value below 35 ms within a fruit image and the deformation parameter registered during the Magness-Taylor firmness test. Finally, all $\mathrm{T}_{2}$ maps of mealy apples show a regional variation of contrast which is not shown for non-mealy apples. Significant differences $(F=$ 19.43) between mealy and non-mealy apples are found in the histograms of the $T_{2}$ maps as mealy apples show a skew histogram combined with a "tail" in their high $T_{2}$ extreme which is not shown in the histograms of non-mealy apples. These histogram features are also shown for an apple showing internal breakdown indicating that in mealy apples there is a differential water movement that may precede internal breakdown.
\end{abstract}

Keywords: Apple; Mealiness; Internal breakdown.

\section{INTRODUCTION}

Mealiness is a negative attribute of sensory texture that combines the sensation of a desegregated tissue with the sensation of lack of juiciness. Within a wide project devoted to the study of mealiness in fruits, consumers perception and means for detection, three sensory panels have been trained to assess this characteristic in apples In all three cases, mealiness has been described as a multidimensional sensory descriptor capable of gathering the loss of consistency and of juiciness. Also a Repertory Grid has been carried out on four countries (Belgium, Denmark, Spain and UK) and five languages (Danish. English, French, Flemish, and Spanish) on 120 consumers per country. The result can be summarized by saying that the consumers perceive mealiness in apples as the loss of crispiness, of hardness and of juiciness. Additionally. several instrumental procedures have been tested for mealiness assessment. In this sense, our laboratory has focused its aims in a first stage on performing instrumental tests for assessing some textural descriptors as crispiness. hardness and juiciness. The results obtained within these tests have shown to correlate well with the sensory measurements in apples, ${ }^{1}$ but also have succeeded when trying to generate several texture degradation levels on peaches from which mealiness appears to be the last stage. ${ }^{2}$ At current level a reference instrumental procedure for destructive mealiness assessment has been developed. It has also been confirmed that mealiness does not appear for all fruits at the same time (apples $s^{2}$ and peaches ${ }^{3}$ ) and therefore it is essential to assess mealiness on individual fruits. Thus, the use of non destructive techniques for a precise assessment of mealiness onset is essential.

The magnetic resonance imaging (MRI) together with $x$-ray imaging techniques are the most extended techniques capable of evaluating global internal quality in a non-destructive way. ${ }^{4}$ MRI makes use of the magnetic properties that some atomic nuclei have, especially hydrogen nuclei from water molecules. When placed in a 
magnetic field (such as in an MR instrument). the natural magnetic fields of the lydrogen nuclei re-orient themselves along the strong magnetic field of the scanner. This orientation may be perturbed by exciting those nuclei with a burst of electromagnetic energy (If pulse). As these excited nuclei realign themselves with the scanner's magnetic field. they emit a radiofrequency signal that can be detected by a receiver coil. The nuclei emit different signals depending on their surroundings. The contrast between various stnictures would be poor since tissues do not differ substantially in water content. However, there are numerous properties of the tissue water which can be exploited to provide contrast and these can be used to obtain another useful information.

The work that will be presented in this paper must be considered as the previous test for extracting MRI features for mealiness assessment in apples. We planned to check the reliability on the decrease of the $T_{2}$ values for apples during storage and to search for MRI image features for mealiness assessment in fruit.

\section{MATERIALS AND METHODS}

\section{Selection of Fruits}

Eight apple fnits corresponding to the variety "TopRed" have been tested under mechanical and MRI imaging tecluniques. These fnits correspond to selected samples of a wide experiment for mealiness assessment on 420 fruits. Four apples correspond to a small sample of fruits stored under controlled atmosphere conditions in commercial storage chambers for a 6 month period, while the rest of 4 apples correspond to a small sample of fnits stored under $2^{\circ} \mathrm{C}$ also in commercial chambers for the same period.

After the set of MRI images was performed on the mentioned apples. there was a need for searching apples showing internal breakdown within the wide experiment in order to confirm the MRI features extracted for the fnits originally tested. In this sense four new apples were chosen within a group of the wide experiment where a $60 \%$ of apples showing internal breakdown had been previously detected. For this group only one apple had developed a internal breakdown disorder.

\section{MRI}

MRI experiments were performed on a Bnker Biospec 47/40 (Ettlingen. Germany) spectrometer equipped with actively shielded imaging gradients capable of reaching $100 \mathrm{mT} / \mathrm{m}$ along all axes. The bore size of the magnet is $40 \mathrm{~cm}$. both with gradient stack in place. the bore is reduced to $26 \mathrm{~cm}$. A home-built high pass birdcage coil $(n=8)$ with an inner diameter of $10.4 \mathrm{~cm}$ and a length of $14.0 \mathrm{~cm}$ was constnicted to adapt the coil to the maximum size of our apples.
Apples were imaged as $64 \times 64$ matrices using the standard two dimensional spin echo sequence. One slice and 20 echoes were collected with different echo time, TE. of 8 and $9.5 \mathrm{~ms}$, and a fixed repetition time of 3000 $\mathrm{ms}$. Other acquisition parameters were a $5 \mathrm{~mm}$ slice thickness and a $10-\mathrm{cm}$ field-of-view (FOV). Pulse gains, attenuator and shimming settings were previously adjusted for a 8-cm sphere filled with doped water (copper sulfate. $1 \% \mathrm{w} / \mathrm{w}$ ) and were held constant along the total experiment, in which several apples were imaged under these conditions. Field homogeneity was tested as the lack of change in the images of the spherical phantom before and after the apples recording. For comparison. all images were reconstructed with identical scale factor. The magnitude of these multi-echo images was fitted on a pixel by pixel basis using a two parameter $\left(T_{2}\right.$ and the signal intensity at equilibrium) monoexponential function without baseline, using the standard Bruker imaging Fit Package, which uses the Levenberg-Marquart criterion for chi-square minimization operating on a Silicon Graphics Indy computer. The software package allows to choose a threshold to remove those pixels with low signal to noise ratio. in such a way that $T_{2}$ maps of all samples were obtained discharging pixels (previous to the fitting) under the $15 \%$ of the maximum signal intensity (this value was found correct to get rid of background pixels). Measurements with the phantom leaded to an accuracy in the $T_{2}$ values assessment of $\pm 2.4 \%$.

Histograms were attained by removing those pixels with $\mathrm{T}_{2}$ values (digitized at four bits) equal to zero (those pixels which did not fit corresponding to core and background areas).

\section{Mechanical Tests}

The mechanical tests carried out on the samples can be summarized as:

- Magness-Taylor penetration test: It was carried out with a Texture Analyser $\mathrm{XT}_{2}$ (Stable Micro Systems, Surrey. UK) on entire. Magness-Taylor flesh penetration test was performed for an 8-1mm diameter rod. A maximum penetration of $8 \mathrm{~mm}$ was applied at 20 $\mathrm{mm} / \mathrm{min}$ speed rate. The maximum penetration force was registered and will be used as Magness-Taylor firmness.

- Confined compression test: It was carried out with the same Texture machine on cylindrical probes of $1.7-\mathrm{cm}$ height and $1.7 \mathrm{~cm}$ of diameter. Probes were confined in a disc of $1.7-\mathrm{cm}$ height. with a hole of the same diameter as the probe. A maximum deformation of 2.5 $\mathrm{mm}$ was applied at $20 \mathrm{~mm} / \mathrm{min}$ speed rate. The rod used in this test had a $15.3-\mathrm{mm}$ diameter in order to avoid rod/disc contacts during compression. Deforma- 


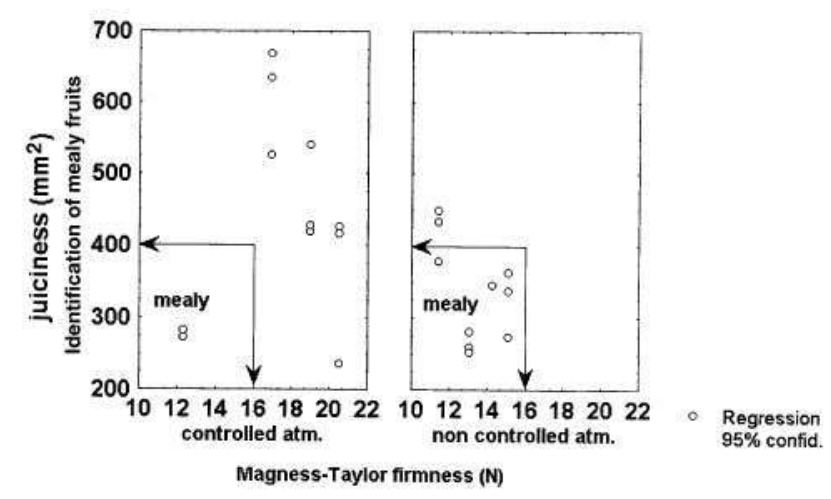

Fig. 1. Identification of mealy fruits through the destructive reference tests used in this work: Magness-Taylor firmness \& confined compression juiciness.

tion was immediately removed at the same speed rate; three repetition was made per fruit. The following parameters were registered through this tests: Force/ deformation ratio within the elastic behavior $(n / \mathrm{mm}$, this magnitude will be used as compression hardness), and the Juice area $\left(\mathrm{mm}^{2}\right)$ of the spot accumulated in a filter paper placed underneath the probe during the test (this magnitude will be used as compression juiciness).

The solid soluble content was measured using a digital refractometer (PR-101 Atago, Atago Co Ltd, Japan).

\section{Statistical Analysis}

Statistica 4.5 for Windows has been used for data analysis purposes.

\section{RESULTS}

\section{General Overview of the Samples}

All the fruits were submitted to controlled atmosphere showed higher soluble solids content, around $13^{\circ} \mathrm{BRIX}$, when compared to the fruit stores under $2^{\circ} \mathrm{C}$ (lower than $12^{\circ}$ BRIX).

The Magness-Taylor Firmness test shows than three out of the four fruits corresponding to the controlled atmosphere sample had a firmness stage above $16 \mathrm{~N}$, while all the fruits stored under $2^{\circ} \mathrm{C}$ had firmness values below $16 \mathrm{~N}$.

\section{Identification of Mealy Fruits by Mechanical Means}

Confined compression test was used for the identification of mealy fruits by destructive instrumental means. This test indicates that only one out of the four fruits tested submitted to controlled atmosphere conditions had developed mealiness, while three out of the four fruits tested stored under $2^{\circ} \mathrm{C}$ had already developed mealiness at that stage (see Fig. 1). In this figure, each three dots of the confined compression test represents one fruit (see Material and Methods).

The classification of the fruits into: Hard (MagnessTaylor Firmness above 16N) and Soft (Magness-Taylor Firmness below $16 \mathrm{~N}$ ), or into non-mealy (hard or soft combined with juiciness above $400 \mathrm{~mm}^{2}$ ) and mealy (soft combined with juiciness below $400 \mathrm{~mm}^{2}$ ) has been used to extract features from the MRI images.

\section{Extraction of Features within the MRI Images for Mealiness Assessment}

From each MRI image corresponding to the nine fruits tested, several characteristic parameters, such as the minimum $T_{2}$ value, the average $T_{2}$ value, and the maximum $T_{2}$ value were extracted (see Table 1) and studied in relation to the classification of the fruits into hard/soft and non-mealy/mealy. There is no significant effect of Hard/Soft Magness-Taylor Firmness on the characteristic $T_{2}$ parameters, but there is a significant effect $(1 \%$ significant level, $F=13.21)$ for the mealy textured fruits on the minimum $\mathrm{T}_{2}$ value (lower mini-

Table 1. Comparison of $\mathrm{T}_{2}$ values in MRI images between non-mealy and mealy apples. The average and standard deviation are plotted for each parameter

\begin{tabular}{|c|c|c|c|c|c|c|c|}
\hline \multirow[b]{2}{*}{$\begin{array}{l}\text { Type of } \\
\text { texture }\end{array}$} & \multirow[b]{2}{*}{$n$} & \multicolumn{5}{|c|}{ Extracted from $T_{2}$ maps } & \multirow{2}{*}{$\begin{array}{c}\begin{array}{c}\text { Extracted from } \\
\text { histograms }\end{array} \\
\begin{array}{c}\text { highest negative } \\
\text { slope }\end{array}\end{array}$} \\
\hline & & $\underset{(\mathrm{ms})}{\min . \mathrm{T}_{2}}$ & $\begin{array}{c}\text { avg. } \mathrm{T}_{2} \\
(\mathrm{~ms})\end{array}$ & $\underset{(\mathrm{ms})}{\max .} \mathrm{T}_{2}$ & $\begin{array}{l}\text { pixels } \\
<30 \mathrm{~ms}\end{array}$ & $\begin{array}{l}\text { pixels } \\
<35 \mathrm{~ms}\end{array}$ & \\
\hline \multirow[t]{2}{*}{ Non-mealy } & 4 & 22.98 & 50.98 & 74.66 & 11.00 & 37.50 & -20.91 \\
\hline & & +3.57 & +2.50 & +4.68 & +6.48 & +12.88 & +2.85 \\
\hline \multirow[t]{2}{*}{ Mealy } & 4 & 16.28 & 51.44 & 76.35 & 16.75 & 38.25 & -30.00 \\
\hline & & +5.29 & +1.05 & +4.52 & +6.58 & +11.01 & +6.66 \\
\hline ANOVA & & $* *$ & ns & ns & * & ns & $* *$ \\
\hline$F$ value & & 13.21 & & & 4.65 & & 19.43 \\
\hline
\end{tabular}

${ }^{* *}$ means $p<1 \%$ and $* p<5 \%$. 
mum $T_{2}$ for mealy fruits). In order to check the consistency of the minimum $T_{2}$ value in relation to the whole image, a study on the number of pixels with a $T_{2}$ value below $30 \mathrm{~ms}$ or $35 \mathrm{~ms}$ (intermediate values between the minimum and the average $T_{2}$ values) was carried out. The number of pixels below $30 \mathrm{~ms}$ represented less than $1 \%$ of the fruit (in all cases around 2000 pixels). while the number of pixels below $35 \mathrm{~ms}$ represented around a $2 \%$ of the fnit. The effect of the non-mealy/mealy characteristic of the fruit remains significant for the number of pixels below $30 \mathrm{~ms}$ ( $5 \%$ significant level, $F=4.65$ ) while it turns to non significant for the number of pixels below $35 \mathrm{~ms}$, as it is also non significant for the average $T_{2}$ value or for the maximum $T_{2}$ value

A study on the correlation coefficients has been made between the mechanical and the MRI parameters. The results show that only the deformation parameter measured during the Magness-Taylor Firmness test shows a significant correlation with some of the MRI parameters: the number of pixels below $30 \mathrm{~ms}(\mathrm{r}=-0.71)$ and the number of pixels below $35 \mathrm{~ms}(r=-0.76)$.

Apart from the study on several characteristic $T_{2}$ parameters. further work has been carried out on the histograms of the fruits as a visual stidy of the images indicated the presence of high/low $T_{2}$ contrast areas by the core of mealy apples similar to the ones obtained for apples with internal breakdown. The study carried out on the histograms (see Figs. 2 and 3 ) revealed that normal distributions of the $T_{2}$ values are found for all the NonMealy apples. while all the Mealy apples show more skew histograms. This fact can be mathematically demonstrated by a significant difference in the negative slope of the histogram (see Table 1). The histograms corresponding to mealy apples also show tailed histograms by the high $T_{2}$ extreme similar to the tail observed in an histogram of an apple with internal breakdown (see Fig. 4). The high/low $T_{2}$ contrast areas found for the Mealy fnits indicates the presence of water in a motional way that may precede internal breakdown of tissues. The proportion of pixels involved in the tails of the histograms for the Mealy apples (around 14\%) is similar to the proportion of pixels involved in the tail of a watercored apple $(16 \%)$. The observation of a tailed histogram allows to segment the image for the assessment of the tissue area were the water appears in a motional way.

\section{DISCUSSION}

In the field of internal quality evaluation. the MRI imaging teclunique has been proposed to identify mechanical damages (bruises) in onions, apples. peaches and pears. ${ }^{5}$ The MRI imaging technique has also been used to assess internal injury due to field conditions: water-core in apples, ${ }^{6}$ and storage treatments as: chilling injury in persimmons. ${ }^{7}$ and internal breakdown in melons. $^{8}$

In the case of persimmons, the chilling injury is described as a initial tissue breakdown and lack of cohesion between cells followed by formation of a firm gel and by a lack of juiciness without changes in the total amount of water content. Also a browning of the flesh is indicated. ${ }^{7}$ This definition fits into the previous description of mealiness. Also in this study an indication on the differential pattern on the injury onset is given, as the chilling injury affects only some fruits and not the whole of them. This fact also agrees with the mealiness onset pattern given for apples by Harker and Hallet $t^{3}$ and for peaches by Ortiz et al. ${ }^{2}$

The study of Clark and Forbes ${ }^{7}$ also indicates that for some bagged replicates it appeared a very bright area by the core which indicated the presence of water in a motional way. The fruits showing this bright contrast area also showed the presence of the mentioned chilling injury through destructive test for gelling, juiciness and firmness assessments, which was undetectable else nondestructive way. In control samples, this bright contrast areas in the core of the persimmons tended to evolve into internal browning. This showed that the internal browning and the above defined chilling injury could be related. Finally the same study indicates that the only quantitative difference in the MRI images of persimmons before and after a six weeks of cold storage period is a slight decrease in the $T_{2}$ values which the authors of this study assume as an effect of the loss of fresh weight (water) during storage. ${ }^{7}$

The studies on water-core in apples ${ }^{6}$ and internal breakdown in melons ${ }^{8}$ show that both disorders have a very similar MRI feature corresponding, as in the case of persimmons, to very bright/dark areas by the core of the fnit (water in a motional way). However, the main visual characteristic of water-cored apples is a translucent appearance of the flesh while melons showing internal breakdown have a dark brown appearance as well as tissue degradation associated with over-ripening and senescence. ${ }^{9}$ The study of Wang et $\mathrm{al}^{6}{ }^{6}$ indicates that water-core in apples remains in a determined area ( $\pm 20 \%$ from the equator of the fruit toward the calyx or to the steam) while internal breakdown may extend along the flesh to affect most of the calyx-steam axis. ${ }^{9}$

\section{CONCLUSIONS}

The conclusions obtained for the previous test on the extraction of MRI image features for mealiness assessment in apples can be summarized as:

1. The minimum $\mathbf{T}_{2}$ value is the only $\mathbf{T}_{2}$-related parameter that shows a significant effect of the non-mealy/ mealy texture stage of the fruit. 

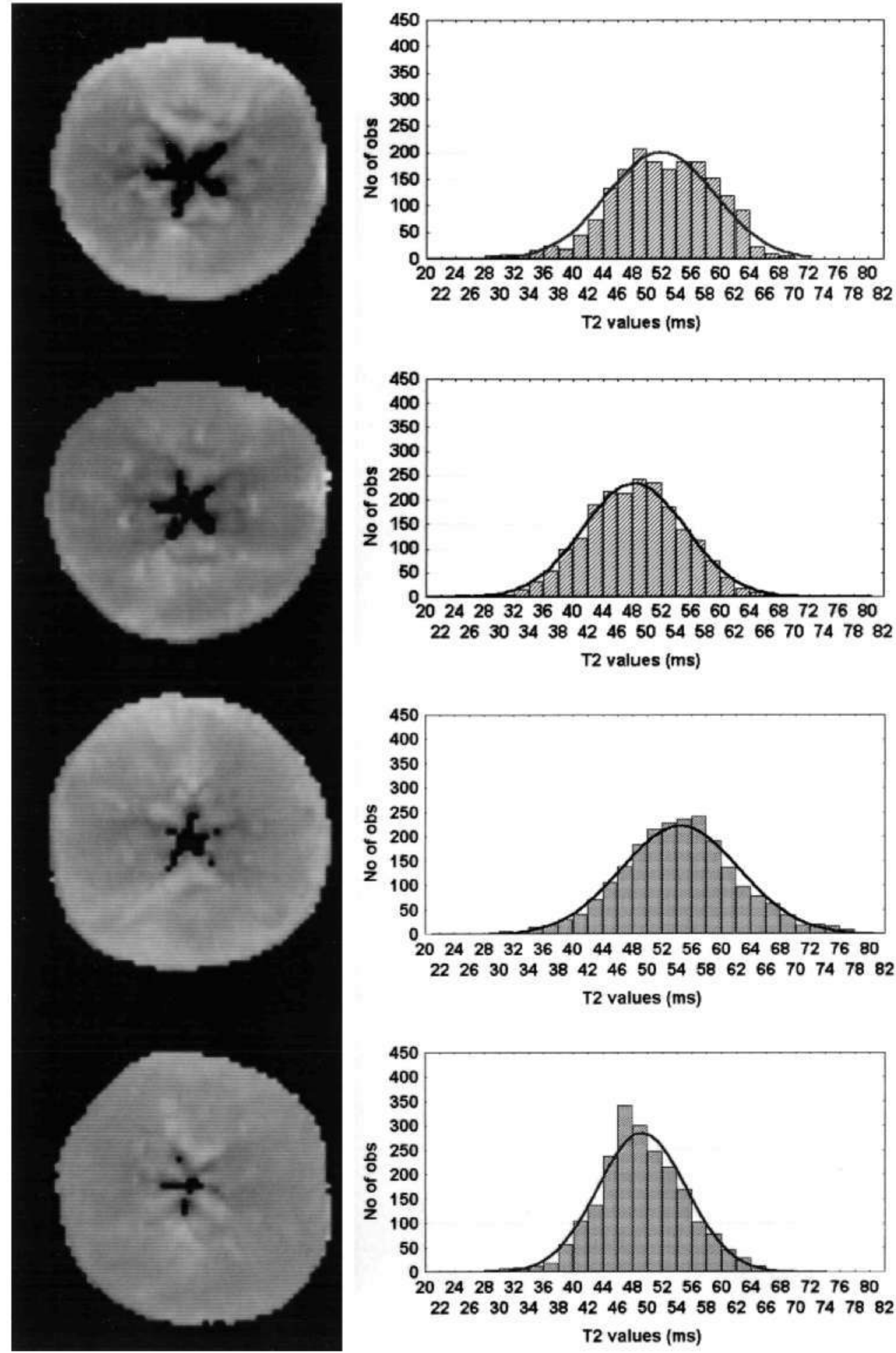

Fig. 2. $T_{2}$ images and histograms corresponding to the four non-mealy fruits tested. Note that $T_{2}$ values follow normal distributions.

2. The number of pixels into a $T_{2}$ image below $35 \mathrm{~ms}$ (an intermediate value between the minimum and the average $T_{2}$ values) shows a significant correlation coefficient $(\mathrm{r}=-0.76)$ with the deforma- tion parameter registered within the MagnessTaylor firmness test, though this MRI parameter only represents a $2 \%$ of the cross-section of the fruit. 

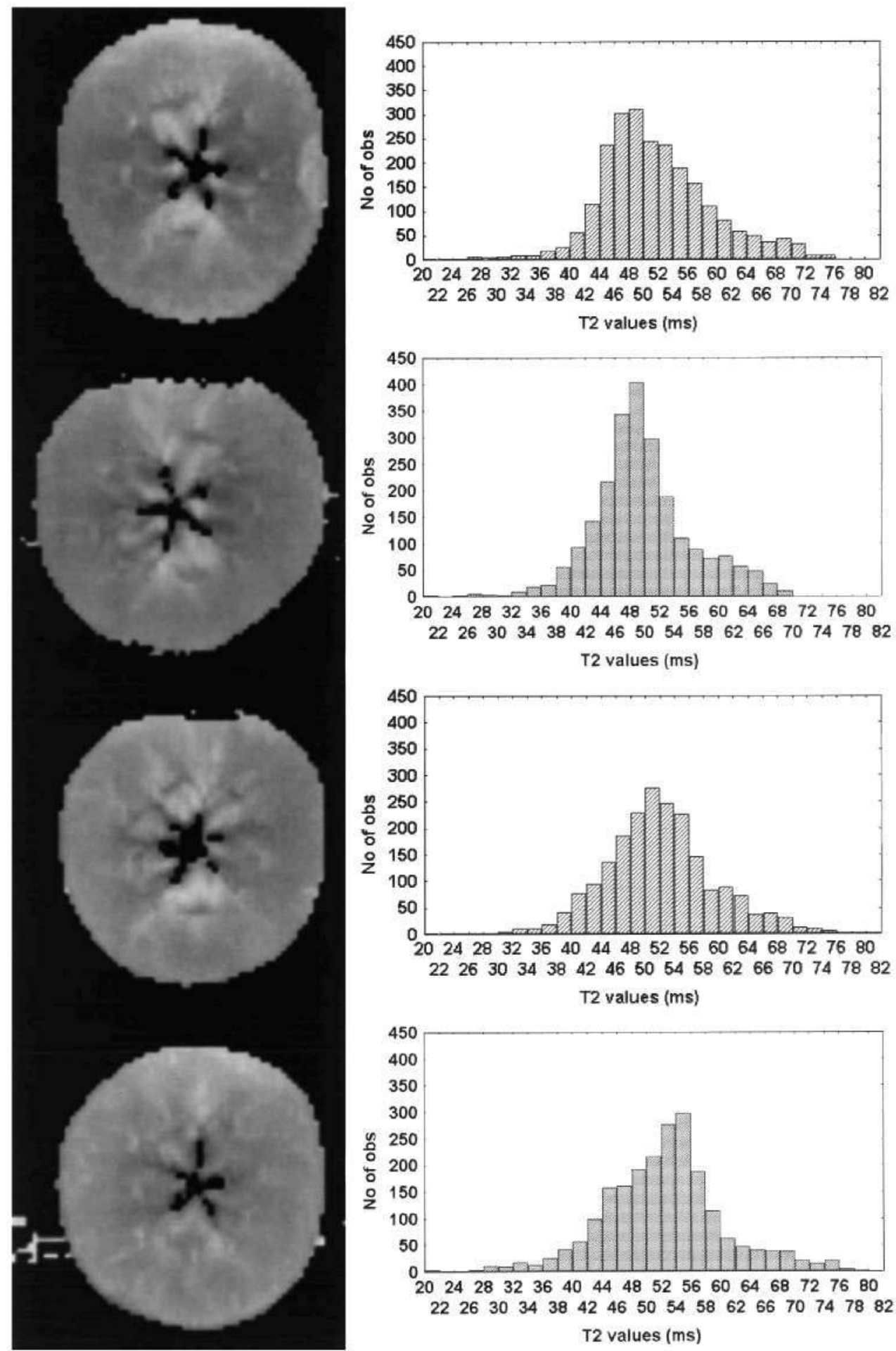

Fig. 3. $T_{2}$ maps and histograms corresponding to the four mealy fruits tested. Note skew histograms and tails by the high $T_{2}$ extreme.

3. The most important MRI feature of mealy apples is the presence of skew histograms with significant differences in their negative slopes $(F=19.43)$ when compared to non-mealy fruits. Also, the his- tograms of mealy apples show a "tail" located in the maximum $\mathrm{T}_{2}$ extreme of the histograms.

4. The shape of the "tailed" histograms of mealy fruits is similar to that of the histogram of an apple with 

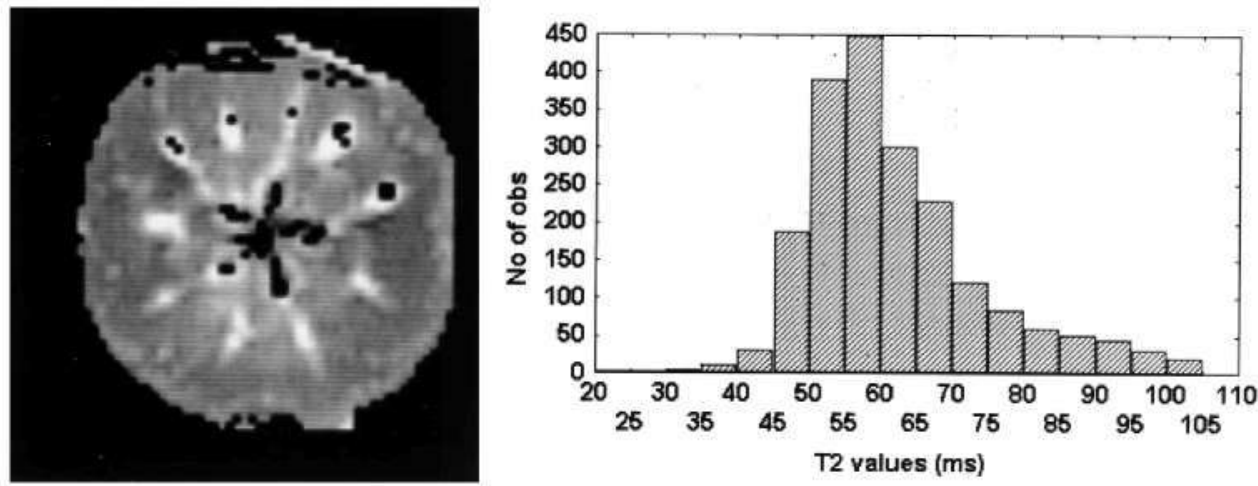

Fig. 4. $\mathrm{T}_{2}$ image and histogram corresponding to an apple showing internal breakdown. Note that the distribution is similar to those found in Fig. 3.

internal breakdown. Moreover, the amount of pixels involved in the tails of the histograms is similar for mealy apples (around 15\%) and for the apple showing internal breakdown (16\%).

Acknowledgments - PBE and MRA acknowledges to the EC Project FAIR CT960302. MEFV and JRC thanks the Spanish Comisi-n Interministerial de Ciencia y Tecnologia (CICYT) for funding (PB91-0368 and $\mathrm{BIO} 97-0543)$.

\section{REFERENCES}

1. Barreiro, P.; M. Ruiz-Altisent, M.; Ortiz, C.; De Smedt, V.; Schotte, S.; Bhanji, Z.; Wakeling, Z. Comparison between sensorial and instrumental measurements for mealiness assessment in apples. A collaborative test. Accepted for publication in the J. Texture Studies 29:1998.

2. Ortiz, C; Barreiro, P; Ruiz-Altisent, M; Riquelme, F. Woolliness assessment in peaches. Comparison between human and instrumental procedures and results. In: V. Bellon-Murel (Ed.). Proceedings of From Sensors to Decision Support System in Agriculture, Food Industry and Environment, vol. 2. SENSORAL 98, CEMAGREF Editions, 1998: pp 151-152.
3. Harker, F.R.; Hallett, I.C. Physiological changes associated with development of mealiness of apple fruit during cold storage. Hortscience 27:1291-1294; 1992.

4. Chen, P.; Sun, Z. A review of non-destructive methods for quality evaluation and sorting of agricultural products. J. Agric. Engineering Res. 49:85-98; 1991.

5. Chen, P.; McCarthy, M.J; Kauten, R. NMR for internal quality evaluation of fruits and vegetables. Transactions of the ASAE 32:1747-1753; 1989.

6. Wang S.Y.; Wang, P.C.; Faust, M. non-destructive detection of water-core in apple with nuclear magnetic resonance imaging. Scientia Hortculturae 35:224-234; 1989.

7. Clark, C.J.; Forbes, S.K. Nuclear magnetic resonance Imaging of the development of chilling injury in 'Fuyu' persimmon (Diospyrus Kaki). New Zealand J. of Crop and Horticultural Sci. 22:209-215; 1994.

8. Zion, B. Detection of internal tissue breakdown in galia melons by magnetic resonance methods. Annual report of the institute of agricultural engineering. The Volcani Centre; 1994.

9. Snowdon, A.L. A colur atlas of post-harvest diseases and disorders of fruits. General introduction and fruits. De Wolfe Scientific ISBN. 1:0 7234 0931:1990. 